EUROPEAN JOURNAL OF PURE AND APPLIED MATHEMATICS

Vol. 12, No. 2, 2019, 577-589

ISSN 1307-5543 - www.ejpam.com

Published by New York Business Global

\title{
On the existence of solution to multidimensional third order nonlinear equations
}

\author{
Samed J.Aliyev ${ }^{1, *}$, Arzu Q.Aliyeva ${ }^{2}$, Goncha Z. Abdullayeva ${ }^{1}$ \\ ${ }^{1}$ Department of Mathematics and Methodology of its Teaching, \\ Baku State University, Z.Khalilov str.23, AZ1148, Baku, Azerbaijan \\ 2 Department of Differential Equations, Institute of Mathematics and Mechanics, \\ Azerbaijan National Academy of Sciences, B.Vahabzade str.9, AZ1141, Baku, Azerbaijan
}

\begin{abstract}
In this paper, we prove existence of an almost everywhere solution to mixed problem for a class of third order differential equations by non-zero rotation principle. Also studied the correctness of the formulation of the considered problem.
\end{abstract}

2010 Mathematics Subject Classifications: 35L76, 35L82.

Key Words and Phrases: Nonlinear operator, continuously differentiable, correct formulation.

\section{Introduction}

The paper is devoted to the problem of existence of almost everywhere solution and correctness of the formulation to the following multidimensional mixed problem for the third order nonlinear equation:

$$
\begin{gathered}
\frac{\partial^{2} u(t, x)}{\partial t^{2}}-\frac{\partial}{\partial t} L(u(t, x))=\mathrm{F}(u(t, x)) \quad(t \in[0, T], x \in \Omega), \\
u(0, x)=\varphi(x) \quad(x \in \Omega), \quad u_{t}(0, x)=\psi(x) \quad(x \in \Omega), \\
\left.u(t, x)\right|_{\Gamma}=0,
\end{gathered}
$$

where $0<T<+\infty ; x=\left(x_{1}, \ldots, x_{n}\right), \Omega$ is a bounded $n$ dimensional domain with an enough smooth boundary $S ; \Gamma=[0, T] \times S$;

$$
L(u(t, x))=\sum_{i, j=1}^{n} \frac{\partial}{\partial x_{i}}\left(a_{i j}(x) \frac{\partial u(t, x)}{\partial x_{j}}\right)-a(x) u(t, x),
$$

*Corresponding author.

DOI: https://doi.org/10.29020/nybg.ejpam.v12i2.3382

Email addresses: samed59@bk.ru (Samed J.Aliyev),

arzu.aliyeva@bk.ru (Arzu Q.Aliyeva), a.q.z.41@mail.ru (Goncha Z. Abdullayeva). 
functions $a_{i j}(x)(i, j=\overline{1, n})$ and $a(x)$ are measurable, and bounded in $\Omega$ and satisfy in $\Omega$ the following conditions:

$$
a_{i j}(x)=a_{j i}(x), a(x) \geq 0, \sum_{i, j=1}^{n} a_{i j}(x) \xi_{i} \xi_{j} \geq \alpha \cdot \sum_{i=1}^{n} \xi_{i}^{2} \quad(\alpha=\text { const }>0)
$$

where $\xi_{i}(i=1,2, \ldots, n)$ are arbitrary real numbers; $\varphi, \psi$ are the given functions; $\mathrm{F}$ is some, generally speaking, nonlinear operator, and $u(t, x)$ is a sought function.

Note that the results of this work improve the results of our article [1]. There have been many works devoted to the study of mixed problems for nonlinear third order equations (see $[2,3,5,8,10,12]$ and references therein), where the problem of existence and uniqueness in appropriate spaces, the problem of blow up of solutions and the problems of asymptotic behavior of solutions are studied.

As well as we know the equations considered in previous publications do not cover the class of equations we study.

Considered by us equations appear in modeling dynamical processes, in elasticity theory and in modeling dynamics of shallow water waves (see $[7,11])$.

\section{Auxiliaries}

In what follows we are using the following notations and facts.

1. We denote by $\dot{D}(\Omega)$ the class of all continuously differentiable functions on $\Omega$ which vanished near the boundary of $\Omega$. The closure of $\dot{D}(\Omega)$ with respect to the norm of $W_{2}^{1}(\Omega)$ we denote by $\stackrel{\circ}{D}(\Omega)$. Hence $\stackrel{\circ}{D}(\Omega) \subset W_{2}^{1}(\Omega)$.

Denote $\dot{D}_{1}\left(Q_{T}\right)\left(Q_{T} \equiv[0, T] \times \Omega\right)$ the class of all continuously differentiable functions on the cylinder $Q_{T}$ are equal to zero in the $\delta$ neighborhood of the lateral surface on the cylinder $Q_{T}$, having the form: $Q_{T, \delta} \equiv[0, T] \times \Omega_{\delta}$ where $\Omega_{\delta}$ is a $\delta$ neighborhood of the boundary of $\Omega$. The closure of $\dot{D}_{1}\left(Q_{T}\right)$ with respect to the norm of $W_{2}^{1}\left(Q_{T}\right)$ we denote by $\stackrel{\circ}{D}_{1}\left(Q_{T}\right)$. Hence $\stackrel{\circ}{D}_{1}\left(Q_{T}\right) \subset W_{2}^{1}\left(Q_{T}\right)$.

Definition. The function $u(t, x) \in \stackrel{\circ}{D}_{1}\left(Q_{T}\right)$ belonging to the space $L_{2}\left(Q_{T}\right)$ together with all its derivatives $u_{t}(t, x), u_{x_{i}}(t, x) \quad(i=\overline{1, n}), u_{t x_{i}}(t, x) \quad(i=\overline{1, n}), u_{x_{i} x_{j}}(t, x) \quad(i, j=$ $\overline{1, n}), u_{t t}(t, x), u_{t x_{i} x_{j}}(t, x)(i, j=\overline{1, n})$ satisfying equation (1) almost everywhere in $Q_{T}$ and taking initial values (2) almost everywhere in $\Omega$ is called an almost everywhere solution of the problem (1)-(3).

2. For investigation of the problem (1)-(3) we recall one property of the operator $L$, generating by the differential expression (4) and boundary condition (3): there are denumerable number of negative eigenvalues

$$
0>-\lambda_{1}^{2} \geq-\lambda_{2}^{2} \geq \ldots \geq-\lambda_{s}^{2} \geq \ldots, \quad\left(0<\lambda_{s} \rightarrow+\infty \text { as } s \rightarrow \infty\right)
$$

with the corresponding generalized eigenfunctions $v_{s}(x)$ which are complete and orthonormal in $L_{2}(\Omega)$. We call function $v_{s}(x) \in \stackrel{\circ}{D}(\Omega)$ a generalized eigenfunction of the operator 
$L$, if it is not identically zero and

$$
\int_{\Omega}\left\{\sum_{i, j=1}^{n} a_{i j}(x) \frac{\partial v_{s}(x)}{\partial x_{i}} \cdot \frac{\partial \Phi(x)}{\partial x_{j}}+a(x) v_{s}(x) \Phi(x)\right\} d x=\lambda_{s}^{2} \int_{\Omega} v_{s}(x) \Phi(x) d x
$$

for any function $\Phi(x) \in \stackrel{\circ}{D}(\Omega)$.

As the system $\left\{v_{s}(x)\right\}_{s=1}^{\infty}$ is complete orthonormal in $L_{2}(\Omega)$, then it is evident that every almost everywhere solution of problem (1)-(3) has the following form:

$$
u(t, x)=\sum_{s=1}^{\infty} u_{s}(t) v_{s}(x)
$$

where

$$
u_{s}(t)=\int_{\Omega} u(t, x) v_{s}(x) d x \quad(s=1,2, \ldots) .
$$

Then, after applying the Fourier method, finding the unknown Fourier coefficients $u_{s}(t)(s=1,2, \ldots)$ for the almost everywhere solution $u(t, x)$ of the problem (1)-(3) is reduced to the solution of the following countable system of nonlinear integro-differential equations:

$$
\begin{gathered}
u_{s}(t)=\varphi_{s}+\frac{1}{\lambda_{s}^{2}}\left(1-e^{-\lambda_{s}^{2} t}\right) \psi_{s} \\
+\frac{1}{\lambda_{s}^{2}} \int_{0}^{t} \int_{\Omega} \mathrm{F}(u(\tau, x)) \cdot\left[1-e^{-\lambda_{s}^{2}(t-\tau)}\right] v_{s}(x) d x d \tau \quad(s=1,2, \ldots ; t \in[0, T]),
\end{gathered}
$$

where

$$
\varphi_{s}=\int_{\Omega} \varphi(x) v_{s}(x) d x, \quad \psi_{s}=\int_{\Omega} \psi(x) v_{s}(x) d x \quad(s=1,2, \ldots) .
$$

Proceeding from the definition of almost every where solution of problem (1)-(3), it is easy to prove (see [1]) the following

Lemma. If $u(t, x)=\sum_{s=1}^{\infty} u_{s}(t) v_{s}(x)$ is any almost everywhere solution of problem (1)-(3) and the generalized derivatives $\frac{\partial}{\partial x_{k}} a_{i j}(x) \quad(i, j, k=1,2, \ldots, n)$ are bounded on $\Omega$, then functions $u_{s}(t)(s=1,2, \ldots)$ satisfy system $(5)$.

3. We denote by $B_{\beta_{0}, \ldots, \beta_{l}, T}^{\alpha_{0}, \ldots, \alpha_{l}}$ a totality of all the functions of the from

$$
u(t, x)=\sum_{s=1}^{\infty} u_{s}(t) v_{s}(x)
$$


considered in $Q_{T}=[0, T] \times \Omega$, where $u_{s}(t) \in C^{(l)}([0, T])$ for all $s$ and

$$
N_{T}(u) \equiv \sum_{i=0}^{l}\left\{\sum_{s=1}^{\infty}\left(\lambda_{s}^{\alpha_{i}} \cdot \max _{0 \leq t \leq T}\left|u_{s}^{(i)}(t)\right|\right)^{\beta_{i}}\right\}^{\frac{1}{\beta_{i}}}<+\infty,
$$

with $\alpha_{i} \geq 0, \quad 1 \leq \beta_{i} \leq 2 \quad(i=0,1, \ldots, n)$. We define the norm in this set as $\|u\|=N_{T}(u)$. It is evident that all these spaces are Banach spaces ([6, p.50]).

4. Let $G$ be class all functions $u(t, x)$ which have the properties

$$
\begin{gathered}
u(t, x), u_{t}(t, x), u_{x_{i}}(t, x) \quad(i=\overline{1, n}), u_{t x_{i}}(t, x)(i=\overline{1, n}), u_{x_{i} x_{j}}(t, x) \quad(i, j=\overline{1, n}), u_{t t}(t, x), \\
u_{t x_{i} x_{j}}(t, x)(i, j=\overline{1, n}) \in L_{2}\left(Q_{T}\right) .
\end{gathered}
$$

\section{On the existence of almost everywhere solution}

In this section, using non-zero rotation principle, the following existence theorem for the almost everywhere solution of problem (1)-(3) is proved for $n$ :

Theorem 1. Let

(i) $a_{i j}(x) \in C^{(2)}(\bar{\Omega}) \quad(i, j=\overline{1, n}) ; a(x) \in C^{(1)}(\bar{\Omega}) ; S \in C^{(3)}$; the eigenfunctions $v_{s}(x)$ of the operator $L$ under boundary condition $\left.v_{s}(x)\right|_{S}=0$ be three times continuously differentiable on $\bar{\Omega} ; \varphi(x) \in W_{2}^{3}(\Omega) ; \varphi(x), L \varphi(x) \in \stackrel{\circ}{D}(\Omega) ; \psi(x) \in W_{2}^{2}(\Omega) \cap \stackrel{\circ}{D}(\Omega)$.

2. $\mathrm{F}=\mathrm{F}_{1}+\mathrm{F}_{2}+\mathrm{F}_{3}$, where

a) the operator $\mathrm{F}_{1}$ acts from the $B_{2, T}^{2}$ into the space $W_{t, x, 2}^{0,1}\left(Q_{T}\right)$ continuously and for all $u \in B_{2, T}^{2}, t \in[0, T]$ :

$$
\left\|\mathrm{F}_{1}(u(t, x))\right\|_{W_{2}^{1}(\Omega)} \leq a_{1}(t)+a_{2}(t) \cdot\|u\|_{B_{2, T}^{2}}^{\gamma}+a_{3}(t) \cdot\|u\|_{B_{2, t}^{2}},
$$

where $a_{i}(t) \in L_{2}(0, T)(i=1,2,3)$ and $0<\gamma<1$;

b) the operator $\mathrm{F}_{2}$ acts from the closed ball $K^{*}\left(\|u\|_{B_{2, T}^{2}} \leq \frac{1}{\lambda_{1}} a\right)$ into the space $W_{t, x, 2}^{0,1}\left(Q_{T}\right)$ continuously, where

$$
\begin{gathered}
a>a_{0} \equiv \max \left\{y: y^{2} \leq\left(A_{1}+A_{2}|y|^{2 \gamma}\right) \cdot A_{3}\right\}, \\
A_{1} \equiv 2\|w(t, x)\|_{B_{2,2, T}^{3,2}}^{2}+6(2 T+1) \cdot C_{0}^{2} \cdot\left\|a_{1}(t)\right\|_{L_{2}(0, T)}^{2}, \\
A_{2} \equiv 6(2 T+1) \cdot C_{0}^{2} \cdot \frac{1}{\lambda_{1}^{2 \gamma}} \cdot\left\|a_{2}(t)\right\|_{L_{2}(0, T)}^{2},
\end{gathered}
$$




$$
\begin{gathered}
A_{3} \equiv \exp \left\{6(2 T+1) \cdot C_{0}^{2} \cdot \frac{1}{\lambda_{1}^{2}} \cdot\left\|a_{3}(t)\right\|_{L_{2}(0, T)}^{2}\right\}, \\
w(t, x)=\sum_{s=1}^{\infty}\left\{\varphi_{s}+\frac{1}{\lambda_{s}^{2}}\left[1-e^{-\lambda_{s}^{2} t}\right] \psi_{s}\right\} \cdot v_{s}(x), \\
C_{0} \equiv \max \left\{n \cdot \max _{i, j=\overline{1, n}}\left\{\left\|a_{i j}(x)\right\|_{C(\bar{\Omega})}\right\},\|a(x)\|_{C(\bar{\Omega})}\right\}^{\frac{1}{2}} ;
\end{gathered}
$$

c)

$$
\inf _{u \in M}\left\{\left\|u-Q_{1}(u)\right\|_{B_{2,2, T}^{3,2}}-\left\|Q_{2}(u)\right\|_{B_{2,2, T}^{3,2}}\right\}>0
$$

where $M$ the boundary of the ball $K\left(\|u\|_{B_{2,2, T}^{3,2}} \leq a\right), \quad Q_{1}(u)=w+P\left(\mathrm{~F}_{1}(u)\right)$, $Q_{2}(u)=P\left(\mathrm{~F}_{2}(u)\right)$ and

$$
P(u(t, x)) \equiv \sum_{s=1}^{\infty} \frac{1}{\lambda_{s}^{2}} \int_{0}^{t} \int_{\Omega} u(\tau, \xi) v_{s}(\xi) \cdot\left[1-e^{-\lambda_{s}^{2}(t-\tau)}\right] d \xi d \tau \cdot v_{s}(x) ;
$$

d) the operator $\mathrm{F}_{3}$ acts from the closed ball $K_{\rho}\left(\|u\|_{B_{2,2, T}^{3,2}} \leq \rho\right)$ into the space $W_{t, x, 2}^{0,1}\left(Q_{T}\right)$ and for all $u, v \in K_{\rho}$ :

$$
\left\|\mathrm{F}_{3}(u)-\mathrm{F}_{3}(v)\right\|_{W_{t, x, 2}^{0,1}\left(Q_{T}\right)} \leq q \cdot\|u-v\|_{B_{2,2, T}^{3,2}}
$$

where

$$
\begin{gathered}
\rho \geq a, \rho \geq \rho_{0} \equiv \sup _{u \in K}\left\{\left\|Q_{1}(u)+Q_{2}(u)\right\|_{B_{2,2, T}^{3,2}}\right\}, \\
\left(\sqrt{T}+\frac{1}{\sqrt{2}}\right) \cdot C_{0} \cdot q \equiv q_{0} \leq 1-\frac{\rho_{0}}{\rho}, \quad q_{0}<1 ;
\end{gathered}
$$

e)

$$
\inf _{u \in M}\left\{\left\|u-Q_{1}(u)-Q_{2}(u)\right\|_{B_{2,2, T}^{3,2}}-\left\|Q_{3}(u)\right\|_{B_{2,2, T}^{3,2}}\right\}>0, \quad Q_{3}(u)=P\left(\mathrm{~F}_{3}(u)\right) ;
$$

f) $\mathrm{F}_{3}(0)=0$.

3. a) For any $u \in B_{2,2, T}^{3,2}$ for almost all $t \in[0, T], \mathrm{F}_{1}(u(t, x)) \in \stackrel{\circ}{D}(\Omega)$;

b) For any $u \in K$ for almost all $t \in[0, T], \mathrm{F}_{2}(u(t, x)) \in \stackrel{\circ}{D}(\Omega)$;

c) For any $u \in K_{\rho}$ for almost all $t \in[0, T], \mathrm{F}_{3}(u(t, x)) \in \stackrel{\circ}{D}(\Omega)$.

Then problem (1)-(3) has an almost everywhere solution. 
Proof. Using condition 3 of this theorem, we have

$$
\begin{gathered}
Q_{1}(u(t, x))=w(t, x)+\sum_{s=1}^{\infty} \frac{1}{\lambda_{s}^{3}} \int_{0}^{t} \int_{\Omega}\left[\sum_{i, j=1}^{n} a_{i j}(\xi) \frac{\partial}{\partial \xi_{i}} \mathrm{~F}_{1}(u(\tau, \xi)) \cdot \frac{\partial}{\partial \xi_{j}}\left(\frac{v_{s}(\xi)}{\lambda_{s}}\right)\right. \\
\left.+a(\xi) \mathrm{F}_{1}(u(\tau, \xi)) \cdot \frac{v_{s}(\xi)}{\lambda_{s}}\right] \cdot\left[1-e^{-\lambda_{s}^{2}(t-\tau)}\right] d \xi d \tau \cdot v_{s}(x) \quad \forall u \in B_{2,2, T}^{3,2} \\
Q_{2}(u(t, x))=\sum_{s=1}^{\infty} \frac{1}{\lambda_{s}^{3}} \int_{0}^{t} \int_{\Omega}\left[\sum_{i, j=1}^{n} a_{i j}(\xi) \frac{\partial}{\partial \xi_{i}} \mathrm{~F}_{2}(u(\tau, \xi)) \cdot \frac{\partial}{\partial \xi_{j}}\left(\frac{v_{s}(\xi)}{\lambda_{s}}\right)\right. \\
\left.+a(\xi) \mathrm{F}_{2}(u(\tau, \xi)) \cdot \frac{v_{s}(\xi)}{\lambda_{s}}\right] \cdot\left[1-e^{-\lambda_{s}^{2}(t-\tau)}\right] d \xi d \tau \cdot v_{s}(x) \quad \forall u \in K \\
Q_{3}(u(t, x))=\sum_{s=1}^{\infty} \frac{1}{\lambda_{s}^{3}} \int_{0}^{t} \int_{\Omega}\left[\sum_{i, j=1}^{n} a_{i j}(\xi) \frac{\partial}{\partial \xi_{i}} \mathrm{~F}_{3}(u(\tau, \xi)) \cdot \frac{\partial}{\partial \xi_{j}}\left(\frac{v_{s}(\xi)}{\lambda_{s}}\right)\right. \\
\left.+a(\xi) \mathrm{F}_{3}(u(\tau, \xi)) \cdot \frac{v_{s}(\xi)}{\lambda_{s}}\right] \cdot\left[1-e^{-\lambda_{s}^{2}(t-\tau)}\right] d \xi d \tau \cdot v_{s}(x) \quad \forall u \in K_{\rho} .
\end{gathered}
$$

It is easy to obtain that, for any $u, v \in B_{2,2, T}^{3,2}$

$$
\begin{gathered}
\left\|Q_{1}(u)-Q_{1}(v)\right\|_{B_{2,2, T}^{3,2}} \leq\left(\sqrt{T}+\frac{1}{\sqrt{2}}\right)\left\{\int _ { 0 } ^ { T } \int _ { \Omega } \left[\sum _ { i , j = 1 } ^ { n } a _ { i j } ( \xi ) \frac { \partial } { \partial \xi _ { i } } \left(\mathrm{F}_{1}(u(\tau, \xi))\right.\right.\right. \\
\left.\left.\left.-\mathrm{F}_{1}(v(\tau, \xi))\right) \cdot \frac{\partial}{\partial \xi_{j}}\left(\mathrm{~F}_{1}(u(\tau, \xi))-\mathrm{F}_{1}(v(\tau, \xi))\right)+a(\xi)\left(\mathrm{F}_{1}(u(\tau, \xi))-\mathrm{F}_{1}(v(\tau, \xi))\right)^{2}\right] d \xi d \tau\right\}^{\frac{1}{2}} \\
\leq\left(\sqrt{T}+\frac{1}{\sqrt{2}}\right) \cdot C_{0} \cdot\left\|\mathrm{F}_{1}(u(t, x))-\mathrm{F}_{1}(v(t, x))\right\|_{W_{t, x, 2}^{0,1}\left(Q_{T}\right)} .
\end{gathered}
$$

From (21) by virtue of the condition 2 a this theorem it follows that the operator $Q_{1}$ acts continuously from the $B_{2, T}^{2}$ into $B_{2,2, T}^{3,2}$. Since, the space $B_{2,2, T}^{3,2}$ imbedded into the space $B_{2, T}^{2}$ compactly $\left([6\right.$, Theorem 1.1, p.51] $)$, then the operator $Q_{1}$ acts in the $B_{2,2, T}^{3,2}$ compactly.

We consider in $B_{2,2, T}^{3,2}$ the equations

$$
u=\mu Q_{1}(u) \quad \mu \in[0,1],
$$

and a priori estimate their all the possible solutions $u_{\mu}(t, x)$. Then, using inequality (6) $\forall \mu \in[0,1]$ and $t \in[0, T]$ we have 


$$
\begin{gathered}
\left\|u_{\mu}\right\|_{B_{2,2, t}^{3,2}}^{2}=\left\|\mu Q_{1}\left(u_{\mu}\right)\right\|_{B_{2,2, t}^{3,2}}^{2} \leq\left\|Q_{1}\left(u_{\mu}\right)\right\|_{B_{2,2, t}^{3,2}}^{2} \equiv\left\|w+P\left(\mathrm{~F}_{1}\left(u_{\mu}(t, x)\right)\right)\right\|_{B_{2,2, t}^{3,2}}^{2} \\
\leq 2\|w\|_{B_{2,2, T}^{3,2}}^{2}+2(2 T+1) \cdot C_{0}^{2} \cdot \int_{0}^{t}\left\|\mathrm{~F}_{1}\left(u_{\mu}(\tau, x)\right)\right\|_{W_{2}^{1}(\Omega)}^{2} d \tau \\
\leq 2\|w\|_{B_{2,2, T}^{3,2}}^{2}+6(2 T+1) \cdot C_{0}^{2} \cdot\left\{\left\|a_{1}(t)\right\|_{L_{2}(0, T)}^{2}+\left\|a_{2}(t)\right\|_{L_{2}(0, T)}^{2} \cdot \frac{1}{\lambda_{1}^{2 \gamma}} \cdot\left\|u_{\mu}\right\|_{B_{2,2, T}^{3,2}}^{2 \gamma}\right\} \\
+6(2 T+1) \cdot C_{0}^{2} \cdot \frac{1}{\lambda_{1}^{2}} \int_{0}^{t} a_{3}^{2}(\tau) \cdot\left\|u_{\mu}\right\|_{B_{2,2, \tau}^{3,2}}^{2} d \tau,
\end{gathered}
$$

where $C_{0}$ is defined by (12).

From (23), on applying Bellman's inequality [4, pp. 188,189] and using notations (8)-(10), we obtain that $\forall \mu \in[0,1]$ :

$$
\left\|u_{\mu}\right\|_{B_{2,2, T}^{3,2}}^{2} \leq\left(A_{1}+A_{2} \cdot\left\|u_{\mu}\right\|_{B_{2,2, T}^{3,2}}^{2 \gamma}\right) \cdot A_{3} .
$$

From here, using notation (7), we have

$$
\left\|u_{\mu}(t, x)\right\|_{B_{2,2, T}^{3,2}}^{2} \leq a_{0} \quad \forall \mu \in[0,1],
$$

that is, all the possible solutions $u_{\mu}$ of equations (22) are a priori bounded in $B_{2,2, T}^{3,2}$ and belong to the ball $K_{0}\left(\|u\|_{B_{2,2, T}^{3,2}} \leq a_{0}\right)$.

From (22) and (24) we obtain that $\forall \mu \in[0,1]$ completely continuous vector field $T_{\mu}=J-\mu Q_{1}$ has no zeros on the boundary $M$ of the ball $K\left(\|u\|_{B_{2,2, T}^{3,2}} \leq a\right)$, where $J$ is a unit vector field and $a$ is a number appearing in the condition $2 \mathrm{~b}$ this theorem. Consequently, completely continuous vector fields $T_{0}=J$ and $T_{1}=J-Q_{1}$ are homotopic on the sphere $M$. Then their rotation $\delta$ on $M$ are the same, namely:

$$
\delta\left(J-Q_{1} ; M\right)=\delta(J ; M)=1 .
$$

Now, we consider the operator $Q_{2}$ in the closed ball $K$. Just as the completely continuity of the operator $Q_{1}$ in $B_{2,2, T}^{3,2}$, was shown it is easy to show that the operator $Q_{2}$ acts compactly from $K$ into $B_{2,2, T}^{3,2}$. Further, on the boundary $M$ of the ball $K$ we consider completely continuous vector fields $F_{\lambda}=J-Q_{1}-\lambda Q_{2}, \lambda \in[0,1]$. Due to of the condition $2 \mathrm{~b}$ this theorem $\forall \lambda \in[0,1]$ and $u \in M$ we have

$$
\left\|u-Q_{1}(u)-\lambda Q_{2}(u)\right\|_{B_{2,2, T}^{3,2}} \geq\left\|u-Q_{1}(u)\right\|_{B_{2,2, T}^{3,2}}-\left\|Q_{2}(u)\right\|_{B_{2,2, T}^{3,2}}>0 .
$$


Hence, in particular, it follows that completely continuous vector fields $F_{0}=J-Q_{1}$ and $F_{1}=J-Q_{1}-Q_{2}$ are homotopic on the sphere $M$. Consequently, on $M$ their rotations are equal to:

$$
\delta\left(J-Q_{1}-Q_{2} ; M\right)=\delta\left(J-Q_{1} ; M\right)=\delta(J ; M)=1 .
$$

And now in the ball $K_{\rho}\left(\|u\|_{B_{2,2, T}^{3,2}} \leq \rho\right)$ we consider the operator $Q_{3}$. Similar to (21), $\forall u, v \in K_{\rho}$ we have

$$
\begin{gathered}
\left\|Q_{3}(u)-Q_{3}(v)\right\|_{B_{2,2, T}^{3,2}} \leq\left(\sqrt{T}+\frac{1}{\sqrt{2}}\right) \cdot C_{0} \cdot\left\|\mathrm{F}_{3}(u)-\mathrm{F}_{3}(v)\right\|_{W_{t, x, 2}^{0,1}\left(Q_{T}\right)} \\
\leq\left(\sqrt{T}+\frac{1}{\sqrt{2}}\right) \cdot C_{0} \cdot q \cdot\|u-V\|_{B_{2,2, T}^{3,2}}=q_{0} \cdot\|u-V\|_{B_{2,2, T}^{3,2}} .
\end{gathered}
$$

For each fixed $V_{0} \in K_{\rho_{0}}\left(\|u\|_{B_{2,2, T}^{3,2}} \leq \rho_{0}\right.$ ) (where the number $\rho_{0}$ is defined by (16)) and $\varepsilon \in[0,1]$ in the ball $K_{\rho}$ we consider the following equation

$$
u=\varepsilon \cdot Q_{3}(u)+V_{0} .
$$

Taking advantage fact that

$$
Q_{3}(0)=P\left(\mathrm{~F}_{3}(0)\right)=P(0)=0,
$$

for any $u, u_{1}, u_{2} \in K_{\rho}$ we have

$$
\begin{gathered}
\left\|\varepsilon \cdot Q_{3}(u)+V_{0}\right\|_{B_{2,2, T}^{3,2}} \leq\left\|Q_{3}(u)\right\|_{B_{2,2, T}^{3,2}}+\left\|V_{0}\right\|_{B_{2,2, T}^{3,2}} \\
=\left\|Q_{3}(u)-Q_{3}(0)\right\|_{B_{2,2, T}^{3,2}}+\left\|V_{0}\right\|_{B_{2,2, T}^{3,2}} \leq\left(\sqrt{T}+\frac{1}{\sqrt{2}}\right) \cdot C_{0} \cdot q \cdot\|u\|_{B_{2,2, T}^{3,2}}+\left\|V_{0}\right\|_{B_{2,2, T}^{3,2}} \\
=q_{0} \cdot\|u\|_{B_{2,2, T}^{3,2}}+\left\|V_{0}\right\|_{B_{2,2, T}^{3,2}} \leq q_{0} \cdot \rho+\rho_{0} \leq \rho, \\
\left\|\varepsilon \cdot Q_{3}\left(u_{1}\right)+V_{0}-\left[\varepsilon \cdot Q_{3}\left(u_{2}\right)+V_{0}\right]\right\|_{B_{2,2, T}^{3,2}} \\
\leq\left\|Q_{3}\left(u_{1}\right)-Q_{3}\left(u_{2}\right)\right\|_{B_{2,2, T}^{3,2}} \leq q_{0}\left\|u_{1}-u_{2}\right\|_{B_{2,2, T}^{3,2}} .
\end{gathered}
$$

As $q_{0}<1$, then by virtue of the contracted mappings principle, the operator $A(u)=$ $\varepsilon \cdot Q_{3}(u)+V_{0}$ has a unique fixed point $u_{0}$ in $K_{\rho}$. Comparing to each $V_{0} \in K_{\rho_{0}}$ the unique in $K_{\rho}$ solution $u_{0} \in K_{\rho_{0}}$ of equation (26), we generate some operator $R_{\varepsilon}$, acting from $K_{\rho_{0}}$ into $K_{\rho}$. Next, for any $\varepsilon \in[0,1]$ and $V \in K_{\rho_{0}}$ using notation

$$
R_{\varepsilon}(V) \equiv \varepsilon \cdot Q_{3}\left(R_{\varepsilon}(V)\right)+V,
$$


it is easy to get for any $V_{1}, V_{2} \in K_{\rho_{0}}$

$$
\left\|R_{\varepsilon}\left(V_{1}\right)-R_{\varepsilon}\left(V_{2}\right)\right\|_{B_{2,2, T}^{3,2}} \leq \frac{1}{1-\varepsilon \cdot q_{0}} \cdot\left\|V_{1}-V_{2}\right\|_{B_{2,2, T}^{3,2}} .
$$

Due to notation (16) we have $\left(Q_{1}+Q_{2}\right) K \subset K_{\rho_{0}}$. Consequently, for each $\varepsilon \in[0,1]$ the operator $R_{\varepsilon}$ is defined, in particular, and on $\left(Q_{1}+Q_{2}\right) K$. Due to (27) the operator $R_{\varepsilon}$ satisfies a Lipschitz condition (and therefore continuous) on $\left(Q_{1}+Q_{2}\right) K$. And as, the operators $Q_{1}$ and $Q_{2}$ are completely continuous on $K$, then for each $\varepsilon \in[0,1]$ the operator $R_{\varepsilon}\left(Q_{1}+Q_{2}\right)$ compactly on $K$. Further, due to (17), for any $u \in M$ and $\varepsilon \in[0,1]$ we have

$$
\begin{gathered}
\left\|u-Q_{1}(u)-Q_{2}(u)-\varepsilon \cdot Q_{3}(u)\right\|_{B_{2,2, T}^{3,2}} \\
\geq\left\|u-Q_{1}(u)-Q_{2}(u)\right\|_{B_{2,2, T}^{3,2}}-\left\|Q_{3}(u)\right\|_{B_{2,2, T}^{3,2}}>0 .
\end{gathered}
$$

Consequently, the completely continuous vector field $J-Q_{1}-Q_{2}-\varepsilon \cdot Q_{3}$ for any $\varepsilon \in[0,1]$ does not have zeros on $M$. Then does not have zeros on $M$ same way the completely continuous vector field $J-R_{\varepsilon}\left(Q_{1}+Q_{2}\right)$, because each zero on $M$ field $J-R_{\varepsilon}\left(Q_{1}+Q_{2}\right)$ is zero field $J-Q_{1}-Q_{2}-\varepsilon \cdot Q_{3}$.

Thus, the completely continuous vector fields $J-R_{0}\left(Q_{1}+Q_{2}\right)=J-Q_{1}-Q_{2}$ and $J-R_{1}\left(Q_{1}+Q_{2}\right)$ are homotopic on $M$. Consequently, due to (25) we have

$$
\delta\left(J-R_{1}\left(Q_{1}+Q_{2}\right) ; M\right)=\delta\left(J-Q_{1}-Q_{2} ; M\right)=1 .
$$

Hence, by virtue of the non-zero rotation principle $[9$, p. 207], the completely continuous vector field $J-R_{1}\left(Q_{1}+Q_{2}\right)$ has at least one zero inside the ball of $K$. Since each such zero is a zero of the field $J-Q_{1}-Q_{2}-Q_{3}$, it is thus proved that there exists in $K$ at least one fixed point $u(t, x)$ an operator $Q_{1}+Q_{2}+Q_{3}=Q$. Further, it easy to verify (in absolutely the same way as in the proof of Theorem of [2]), that the function $u(t, x)$ is an almost everywhere solution of problem (1)-(3). The theorem is proved.

\section{Correct formulation of the problem}

In this section, using Bellman's inequality ([4, p. 188-189]), the following theorem on continuous dependence (in a certain sense) on initial functions $\varphi(x), \psi(x)$, and nonlinear operator $\mathrm{F}$ for the almost everywhere solution of problem (1)-(3). Problem (1)-(3) with the data $\tilde{\varphi}, \tilde{\psi}, \tilde{\mathrm{F}}$ we let's name problem $\tilde{A}$.

Theorem 2. Let:

(i) Condition 1 of Theorem 1 be satisfied.

(ii) $\tilde{\varphi}(x) \in W_{2}^{3}(\Omega) ; \tilde{\varphi}(x), L \tilde{\varphi}(x) \in \stackrel{\circ}{D}(\Omega) ; \tilde{\psi}(x) \in W_{2}^{2}(\Omega) \bigcap \stackrel{\circ}{D}(\Omega)$.

(iii) For each $u \in B_{2,2, T}^{3,2} \bigcup\left(G \cap B_{2,2, T}^{2,1}\right)$ for almost all $t \in[0, T], \mathrm{F}(u(t, x)) \in \stackrel{\circ}{D}(\Omega)$. 
(iv) For each $u \in B_{2,2, T}^{3,2}$ for almost all $t \in[0, T], \tilde{\mathrm{F}}(u(t, x)) \in \stackrel{\circ}{D}(\Omega)$.

(v) The operators $\mathrm{F}$ and $\tilde{\mathrm{F}}$ acts from $B_{2,2, T}^{3,2} \cup\left(G \cap B_{2,2, T}^{2,1}\right)$ into $W_{t, x, 2}^{0,1}\left(Q_{T}\right)$ so that, for all $u, v \in B_{2,2, T}^{3,2}$ and $t \in[0, T]$

$$
\begin{gathered}
\|\mathrm{F}(u(t, x))\|_{W_{2}^{1}(\Omega)} \leq a(t)+b(t) \cdot\|u\|_{B_{2,2, T}^{3,2}}, \quad a(t), \quad b(t) \in L_{2}(0, T), \\
\|\mathrm{F}(u(t, x))-\mathrm{F}(v(t, x))\|_{W_{2}^{1}(\Omega)} \leq c(t) \cdot\|u-v\|_{B_{2,2, T}^{3,2}}, \quad c(t) \in L_{2}(0, T), \\
\|\tilde{\mathrm{F}}(u(t, x))-\tilde{\mathrm{F}}(v(t, x))\|_{W_{2}^{1}(\Omega)} \leq \tilde{c}(t) \cdot\|u-v\|_{B_{2,2, T}^{3,2}}, \quad \tilde{c}(t) \in L_{2}(0, T), \\
\sup _{u \in K_{1}}\left\{\|\mathrm{~F}(u(t, x))-\tilde{\mathrm{F}}(u(t, x))\|_{W_{t, x, 2}^{0,1}\left(Q_{T}\right)}\right\} \equiv \varepsilon<+\infty,
\end{gathered}
$$

where

$$
K_{1}=K_{1}\left(\|u\|_{B_{2,2, T}^{3,2}} \leq a_{1}\right)
$$

$a_{1} \equiv\left\{\left[2\|w(t, x)\|_{B_{2,2, T}^{3,2}}^{2}+4(2 T+1) \cdot C_{0}^{2} \cdot\|a(t)\|_{L_{2}(0, T)}^{2}\right] \cdot \exp \left[4(2 T+1) \cdot C_{0}^{2} \cdot\|b(t)\|_{L_{2}(0, T)}^{2}\right]\right\}^{\frac{1}{2}}$,

the function $w(t, x)$ is defined by (11) and the number $C_{0}$ is defined by (12).

Then for the unique almost everywhere solutions $u(t, x)$ and $\tilde{u}(t, x)$ of problems (1)-(3) and $\tilde{A}$, respectively, we have

$$
\begin{aligned}
& \|u(t, x)-\tilde{u}(t, x)\|_{B_{2,2, T}^{3,2}} \leq\left\{\sqrt{3} \cdot C_{0} \cdot\|L(\varphi(x)-\tilde{\varphi}(x))\|_{W_{2}^{1}(\Omega)}\right. \\
& +\sqrt{6} \cdot C_{0} \cdot\|\psi(x)-\tilde{\psi}(x)\|_{W_{2}^{1}(\Omega)}+\sqrt{6} \cdot\|L(\psi(x)-\tilde{\psi}(x))\|_{L_{2}(\Omega)} \\
& \left.+\sqrt{6(2 T+1)} \cdot C_{0} \cdot \varepsilon\right\} \cdot \exp \left\{3(2 T+1) \cdot C_{0}^{2} \cdot\|\tilde{c}(t)\|_{L_{2}(0, T)}^{2}\right\}
\end{aligned}
$$

where the operator $L$ is defined by (4) and the number $C_{0}$ is defined by (12).

Proof: By Theorem 2 from work [1] each of the problems (1)-(3) and $\tilde{A}$ has a unique almost everywhere solution

$$
u(t, x)=\sum_{s=1}^{\infty} u_{s}(t) v_{s}(x) \text { and } \tilde{u}(t, x)=\sum_{s=1}^{\infty} \tilde{u}_{s}(t) v_{s}(x)
$$

respectively, so that $u \in K_{1} \subset B_{2,2, T}^{3,2}, \tilde{u} \in B_{2,2, T}^{3,2}$. Then, by virtue of the lemma in section 2 , the functions $u_{s}(t)(s=1,2, \ldots)$ and $\tilde{u}_{s}(t)(s=1,2, \ldots)$ satisfy system $(5)$, so that for 
$\tilde{u}_{s}(t)(s=1,2, \ldots)$ in the system $(5)$ instead of $\varphi_{s}, \psi_{s}$ and $\mathrm{F}(u)$ need to take $\tilde{\varphi}_{s}, \tilde{\psi}_{s}$ and $\tilde{\mathrm{F}}(u)$, respectively.

Using this fact, from system (5) it is easy to obtain that $\forall t \in[0, T]$ :

$$
\begin{aligned}
& \|u-\tilde{u}\|_{B_{2,2, t}^{3,2}}^{2} \leq 3\left\|\sum_{s=1}^{\infty}\left(\varphi_{s}-\tilde{\varphi}_{s}\right) v_{s}(x)\right\|_{B_{2,2, t}^{3,2}}^{2}+3\left\|\sum_{s=1}^{\infty} \frac{1}{\lambda_{s}^{2}}\left(1-e^{-\lambda_{s}^{2} t}\right)\left(\psi_{s}-\tilde{\psi}_{s}\right) v_{s}(x)\right\|_{B_{2,2, t}^{3,2}}^{2} \\
& +3\left\|\sum_{s=1}^{\infty} \frac{1}{\lambda_{s}^{2}} \int_{0}^{t} \int_{\Omega}[\mathrm{F}(u(\tau, \xi))-\tilde{\mathrm{F}}(\tilde{u}(\tau, \xi))] \cdot\left[1-e^{-\lambda_{s}^{2}(t-\tau)}\right] v_{s}(\xi) d \xi d \tau \cdot v_{s}(x)\right\|_{B_{2,2, t}^{3,2}}^{2} \\
& \leq 3 \sum_{s=1}^{\infty}\left[\lambda_{s}^{3}\left(\varphi_{s}-\tilde{\varphi}_{s}\right)\right]^{2}+6 \sum_{s=1}^{\infty}\left[\lambda_{s}\left(\psi_{s}-\tilde{\psi}_{s}\right)\right]^{2}+6 \sum_{s=1}^{\infty}\left[\lambda_{s}^{2}\left(\psi_{s}-\tilde{\psi}_{s}\right)\right]^{2} \\
& +3(2 T+1) C_{0}^{2} \cdot \int_{0}^{t}\|\mathrm{~F}(u(\tau, x))-\tilde{\mathrm{F}}(\tilde{u}(\tau, x))\|_{W_{2}^{1}(\Omega)}^{2} d \tau \\
& \leq 3 \sum_{s=1}^{\infty}\left[\lambda_{s}^{3}\left(\varphi_{s}-\tilde{\varphi}_{s}\right)\right]^{2}+6 \sum_{s=1}^{\infty}\left[\lambda_{s}\left(\psi_{s}-\tilde{\psi}_{s}\right)\right]^{2}+6 \sum_{s=1}^{\infty}\left[\lambda_{s}^{2}\left(\psi_{s}-\tilde{\psi}_{s}\right)\right]^{2} \\
& +6(2 T+1) \cdot C_{0}^{2} \cdot\left[\int_{0}^{t}\|\mathrm{~F}(u(\tau, x))-\tilde{\mathrm{F}}(u(\tau, x))\|_{W_{2}^{1}(\Omega)}^{2} d \tau\right. \\
& \left.+\int_{0}^{t}\|\tilde{\mathrm{F}}(u(\tau, x))-\tilde{\mathrm{F}}(\tilde{u}(\tau, x))\|_{W_{2}^{1}(\Omega)}^{2} d \tau\right] \\
& \leq 3 \cdot C_{0}^{2} \cdot\|L(\varphi(x)-\tilde{\varphi}(x))\|_{W_{2}^{1}(\Omega)}^{2}+6 \cdot C_{0}^{2} \cdot\|\psi(x)-\tilde{\psi}(x)\|_{W_{2}^{1}(\Omega)}^{2} \\
& +6 \cdot\|L(\psi(x)-\tilde{\psi}(x))\|_{L_{2}(\Omega)}^{2}+6(2 T+1) \cdot C_{0}^{2} \cdot\|\mathrm{F}(u(\tau, x))-\tilde{\mathrm{F}}(u(\tau, x))\|_{W_{t, x, 2}^{0,1}\left(Q_{T}\right)}^{2} \\
& +6(2 T+1) \cdot C_{0}^{2} \cdot \int_{0}^{t} \tilde{c}^{2}(\tau) \cdot\|u-\tilde{u}\|_{B_{2,2, \tau}^{3,2}} d \tau \leq 3 \cdot C_{0}^{2} \cdot\|L(\varphi(x)-\tilde{\varphi}(x))\|_{W_{2}^{1}(\Omega)}^{2} \\
& +6 \cdot C_{0}^{2} \cdot\|\psi(x)-\tilde{\psi}(x)\|_{W_{2}^{1}(\Omega)}^{2}+6 \cdot\|L(\psi(x)-\tilde{\psi}(x))\|_{L_{2}(\Omega)}^{2}+6(2 T+1) \cdot C_{0}^{2} \cdot \varepsilon^{2}
\end{aligned}
$$




$$
+6(2 T+1) \cdot C_{0}^{2} \cdot \int_{0}^{t} \tilde{c}^{2}(\tau) \cdot\|u-\tilde{u}\|_{B_{2,2, \tau}^{3,2}}^{2} d \tau .
$$

From here, on applying Bellman's inequality ([4, p.188, 189]), we obtain the estimate (28).

\section{Acknowledgements}

The authors would like to express their deep gratitude to the editorial team and the anonymous referees of European Journal of Pure and Applied Mathematics, for the careful reading of the manuscript as well as their valuable comments and suggestions which helped to improve the present paper.

\section{References}

[1] S.Aliyev, A.Aliyeva, G.Abdullayeva; The study of a mixed problem for one class of third order differential equations, Advances in Difference Equations 208 (2018), 1-10.

[2] S.Aliyev, A.Aliyeva; On the existence for almost everywhere solution of multidimensional mixed problem for one class third order differential equations with nonlinear operator in the right-hand side, International Journal of Pure and Applied Matematics 115 (3), (2017), 549-560.

[3] S.Aliyev, A.Aliyeva; The study of multidimensional mixed problem for one class of third order semilinear psevdohyperbolic equations, European Journal of Pure and Applied Mathematics 10 (5), (2017), 1078-1091.

[4] E.Beckenbach, R.Bellman; Inequalities, Mir, 1965, 276p. (in Russian).

[5] G.Filippo, S.Marco; Global solutions and finite time blow up for damped semilinear wave equations, Ann. Inst. H. Poincare, Anal. Nonlineaire 23(2), (2006), 185-207.

[6] K.Khudaverdiyev; Multidimensional mixed problem for nonlinear hyperbolic equations, Az. gostekn. University publ. Baku, 2011, 611p. (in Russian).

[7] O. Kosike; Existence of global and bounded solutions for damped sublinear wave equations, J. Math. Univ. Tokushima, 42, (2008), 19-26.

[8] E.M. Mamedov; On stabilization of solution of the third order equation with nonlinear boundary conditions, Proc. Inst. Math. and Mech. Azerb. Acad. Sci., 16, (2002), 7580.

[9] Mathematical reference library; Functional analysis, Nauka, 1964, 424p. (in Russian). 
[10] M.A. Salim; On the decay of solutions for a class of quasilinear hyperbolic equations with nonlinear damping and source terms, Math. Meth. Appl. Sci., 15, (2005), 18191828.

[11] Z. Yang; Cauchy problem for quasilinear wave equations with nonlinear damping and source terms, J. Math. Anal. and Appl., 300(1), (2004), 218-243.

[12] Y. Zhifeng, Q. Dehua; Energy decaying and blow up of solution for a Kirchhoff equation with strong damping, J. Math. Res. And Expo., 29(4), (2009), 707-715. 